\title{
FUNCTIONS OF OPERATORS AND THEIR COMMUTATORS IN PERTURBATION THEORY
}

\author{
YU. B. FARFOROVSKAYA \\ Department of Mathematics, Electrotechnical Institute of Communication \\ Nab. r. Moı̌i 61, 191065 St. Petersburg, Russia
}

\begin{abstract}
This paper shows some directions of perturbation theory for Lipschitz functions of selfadjoint and normal operators, without giving precise proofs. Some of the ideas discussed are explained informally or for the finite-dimensional case. Several unsolved problems are mentioned.
\end{abstract}

Introduction. Let $A$ and $B$ be selfadjoint bounded operators on a separable Hilbert space $H$, and $f$ be a Lip 1 function on the real line $\mathbb{R}$, that is, $|f(t)-f(s)| \leq$ const. $|t-s|$ for $t, s \in \mathbb{R}$.

We are mainly interested in two types of results:

1) estimates of a norm of $f(B)-f(A)$ by the same type norm of $B-A$,

2 ) the same problem for the commutators $f(A) B-B f(A)$ and $A B-B A$.

In this paper we focus attention on the following two ideas:

- using a representation of $f(A)-f(B)$ or of the commutator $[f(A), B]=$ $f(A) B-B f(A)$ by double operator Stieltjes integrals (DOI) ( $(1)$, and

- application of the Kantorovich-Rubinshteŭn norm (K-R norm) (§2).

In $\S 1$ we also discuss M. G. KreĬn's trace formula and related results and in $\S 2$ we deal with relations between the K-R norm and DOI, and with generalizations to normal operators. In $\S 3$ several unsolved problems are mentioned.

In the sequel the spectral measure of the operator $A$ regarded as a point function and as a set function is denoted by $E_{\lambda}$ and $E(\Delta)$, respectively; the spectral measure of $B$ is denoted by $F_{\lambda}$ and $F(\Delta)$. We also use constants $c$ or $C$ with various indices.

1991 Mathematics Subject Classification: 47A55, 47A60, 47B15.

The paper is in final form and no version of it will be published elsewhere. 
1. Double operator integrals (DOI). Let us first look at the finite-dimensional case. Let $A$ and $B$ be Hermitian matrices in the $n$-dimensional space $\mathbb{C}^{n}$. The eigenvalues of $A$ will be denoted by $\left\{\lambda_{i}\right\}$ and the corresponding eigenvectors by $\left\{\varphi_{i}\right\}$. Analogous notations for $B$ are $\left\{\mu_{j}\right\}$ and $\left\{\psi_{j}\right\}$. It is clear that $\lambda_{i}$ and $\mu_{j}$ are real numbers. So $f(A)$ has eigenvectors $\varphi_{i}$ with eigenvalues $f\left(\lambda_{i}\right)$. Thus

$$
\left((f(A)-f(B)) \varphi_{i}, \psi_{j}\right)=\left(f\left(\lambda_{i}\right)-f\left(\mu_{j}\right)\right)\left(\varphi_{i}, \psi_{j}\right) .
$$

Also

$$
\left(A \varphi_{i}, \psi_{j}\right)=\lambda_{i}\left(\varphi_{i}, \psi_{j}\right) \quad \text { and } \quad\left(B \varphi_{i}, \psi_{j}\right)=\mu_{j}\left(\varphi_{i}, \psi_{j}\right)
$$

so assuming that $\lambda_{i} \neq \mu_{j}$, one should obtain

$$
\left(\varphi_{i}, \psi_{j}\right)=\frac{\left((A-B) \varphi_{i}, \psi_{j}\right)}{\lambda_{i}-\mu_{j}} .
$$

By substituting the last formula in (1) we arrive at

$$
\left((f(A)-f(B)) \varphi_{i}, \psi_{j}\right)=\frac{f\left(\lambda_{i}\right)-f\left(\mu_{j}\right)}{\lambda_{i}-\mu_{j}}\left((A-B) \varphi_{i}, \psi_{j}\right) .
$$

On the other hand, let $x, y$ be vectors in $\mathbb{C}^{n}$. They may be represented as $x=\sum_{i} x_{i} \varphi_{i}, y=\sum_{j} y_{j} \psi_{j}$. Applying (3) we get

$$
((f(A)-f(B)) x, y)=\sum_{i, j} \frac{f\left(\lambda_{i}\right)-f\left(\mu_{j}\right)}{\lambda_{i}-\mu_{j}} x_{i} \bar{y}_{j}\left((A-B) \varphi_{i}, \psi_{j}\right) .
$$

If we assume that $x_{i} \varphi_{i}=d E_{\lambda} x, y_{j} \psi_{j}=d F_{\mu} y$, and pass from sums to integrals, we can write the representation

$$
((f(A)-f(B)) x, y)=\iint \frac{f(\lambda)-f(\mu)}{\lambda-\mu}\left(d F_{\mu} T d E_{\lambda} x, y\right)
$$

where $T=A-B$.

Formula (4) can be considered not only in $\mathbb{C}^{n}$, but also in an infinite-dimensional Hilbert space. It leads to the formula

$$
f(A)-f(B)=\iint \frac{f(\lambda)-f(\mu)}{\lambda-\mu} d F_{\mu} T d E_{\lambda}
$$

which should be true in some sense. The works of Birman and Solomyak [3], [4] are devoted to discussing the exact meaning of this formula.

Integrals of this type were introduced by Yu. L. Daletskiŭ and S. G. Krĕn in [9]. The exact formula (3) can be replaced by an approximate one if we disregard the eigenvectors $\psi_{j}$ and the corresponding eigenvalues $\mu_{j}$ :

$$
\left((f(A)-f(B)) \varphi_{i}, \psi_{j}\right) \cong \frac{f\left(\lambda_{i}\right)-f\left(\lambda_{j}\right)}{\lambda_{i}-\lambda_{j}}\left((A-B) \varphi_{i}, \varphi_{j}\right) .
$$

Here $A$ is regarded as a principal matrix and $B$ as being "close" to it. It is interesting that the second order terms of the asymptotics were also found in [9]. 
More exactly (under the notations of [9]), if we set $A=H_{0}, B-A=\varepsilon H_{1}$ and suppose that $f$ has a continuous derivative then

$$
\begin{aligned}
f\left(H_{0}+\varepsilon H_{1}\right)= & f\left(H_{0}\right)+\varepsilon \iint \frac{f(\lambda)-f(\mu)}{\lambda-\mu} d E_{\lambda} H_{1} d E_{\mu} \\
& +\varepsilon \iiint \frac{\frac{f(\lambda)-f(\mu)}{\lambda-\mu}-\frac{f(\mu)-f(\nu)}{\mu-\nu}}{\lambda-\nu} d E_{\lambda} H_{1} d E_{\mu} H_{1} d E_{\nu}+\ldots
\end{aligned}
$$

We think that Besov classes will naturally appear when investigating the second order terms. Notice that functions from Besov classes appeared in the work of Peller who studied the nuclearity of $f(A)-f(B)$ with the help of Hankel operators (see [23], [24], [25]).

Let us return to formula (5).

Birman and Solomyak who studied DOI in detail regarded them as transformers. Part of their results can be simplified as follows:

If $f$ is a differentiable function with derivative of bounded variation and the compact operator $A-B$ belongs to the ideal $\mathfrak{S}_{p}$ with the norm $\left(\sum\left|s_{i}\right|^{p}\right)^{1 / p}$, where the $s_{i}$ are the singular numbers (in our case eigenvalues) of $A-B$, then $f(A)-f(B)$ also belongs to $\mathfrak{S}_{p}$ and

$$
\|f(A)-f(B)\|_{p} \leq c_{p} \operatorname{Var}\left(f^{\prime}\right)\|A-B\|_{p}
$$

where $c_{p}$ is a constant and $1<p<\infty$.

The case $p=1$ is particularly interesting because just this case is connected with the trace formula of M. G. Krel̆n. To be more precise, if $A-B$ is nuclear (i.e. belongs to $\mathfrak{S}_{1}$ ) then the following formula holds for a wide class of functions (the trace being the sum of all eigenvalues):

$$
\operatorname{Tr}[f(A)-f(B)]=\int f^{\prime}(\lambda) \xi(\lambda) d \lambda,
$$

where $\xi(\lambda)$ is the so-called spectral shift function for $A$ and $B$, which does not depend on $f$.

This formula has many applications in scattering theory. We consider the finite-dimensional case.

Let $A$ be a Hermitian matrix with distinct eigenvalues $\lambda_{1}<\ldots<\lambda_{s}$ of respective multiplicities $k_{i}$, so $\sum_{i=1}^{s} k_{i}=n$. Let $\alpha<\lambda_{1}$. Consider the trace $\operatorname{Tr}[f(A)-f(\alpha I)]$, which we denote by $L$. Obviously,

$$
\begin{aligned}
L= & k_{1} f\left(\lambda_{1}\right)+k_{2} f\left(\lambda_{2}\right)+\ldots+k_{s} f\left(\lambda_{s}\right)-n f(\alpha) \\
= & n\left[f\left(\lambda_{1}\right)-f(\alpha)\right]+\left(n-k_{1}\right)\left[f\left(\lambda_{2}\right)-f\left(\lambda_{1}\right)\right]+\ldots \\
& +\left(n-k_{1}-\ldots-k_{s-1}\right)\left[f\left(\lambda_{s}\right)-f\left(\lambda_{s-1}\right)\right] \\
= & \int_{\alpha}^{\lambda_{1}} n f^{\prime}(t) d t+\int_{\lambda_{1}}^{\lambda_{2}}\left(n-k_{1}\right) f^{\prime}(t) d t+\ldots
\end{aligned}
$$




$$
+\int_{\lambda_{s-1}}^{\lambda_{s}}\left(n-k_{1}-\ldots-k_{s-1}\right) f^{\prime}(t) d t .
$$

Thus we can represent $L$ in the form

$$
L=\int f^{\prime}(t) \xi_{1}(t) d t
$$

where $\xi_{1}(t)$ equals the difference between the number of eigenvalues of $\alpha I$ and the number of eigenvalues of $A$ that do not exceed $t$. In other words,

$$
\xi_{1}(t)=\operatorname{Tr}\left(E_{t}^{\prime}-E_{t}\right)
$$

where $E_{t}^{\prime}$ is the spectral measure of the operator $\alpha I$.

Further, suppose we have two matrices $A$ and $B$. For $\alpha$ sufficiently small we get

$$
\begin{aligned}
\operatorname{Tr}[f(A)-f(B)] & =\operatorname{Tr}[f(A)-f(\alpha I)]-\operatorname{Tr}[f(B)-f(\alpha I)] \\
& =\int f^{\prime}(t) \xi_{1}(t) d t-\int f^{\prime}(t) \xi_{2}(t) d t=\int f^{\prime}(t) \xi(t) d t
\end{aligned}
$$

where $\xi_{1}(t)=\operatorname{Tr}\left(E_{t}^{\prime}-E_{t}\right), \xi_{2}(t)=\operatorname{Tr}\left(E_{t}^{\prime}-F_{t}\right)$, and $\xi(t)=\xi_{1}(t)-\xi_{2}(t)=$ $\operatorname{Tr}\left(F_{t}-E_{t}\right)$.

Therefore in the finite-dimensional case the trace formula holds and the spectral shift function for the operators $A$ and $B$ is $\xi(t)=\operatorname{Tr}\left(F_{t}-E_{t}\right)$.

Notice that, as shown by M. G. Kreın [18], the function $\xi(t)$ cannot be defined by the above formula in the case of infinite dimensions because the operator $F_{t}-E_{t}$ may not be nuclear; nevertheless the trace formula does hold in that case for $f$ smooth enough. See [18] for more details.

Note also that in [11] we have constructed an example showing that if $f \in \operatorname{Lip} 1$ then $f(A)-f(B)$ can be a non-nuclear operator for a nuclear perturbation $A-B$. Thus the Lip 1 condition on $f$ is necessary but not sufficient for the operator $f(A)-f(B)$ to be nuclear (and for the trace formula (7) to hold).

The weakest condition on $f$ which guarantees the nuclearity was found by V. V. Peller; it is the membership in the Besov class $B_{\infty, 1}^{1}$. On the other hand, it was shown in [5] that if $f^{\prime} \in \operatorname{Lip} \alpha$ for some $\alpha>0$, then the trace formula (7) holds.

Consider now the commutators $f(A) B-B f(A)$ in terms of DOI.

Let $A, B$ be operators in an $n$-dimensional space. Then obviously

$$
\left((A B-B A) \varphi_{i}, \varphi_{j}\right)=\left(\lambda_{j}-\lambda_{i}\right)\left(B \varphi_{i}, \varphi_{j}\right) .
$$

Hence

$$
\begin{aligned}
\left((f(A) B-B f(A)) \varphi_{i}, \varphi_{j}\right) & =\left(f\left(\lambda_{j}\right)-f\left(\lambda_{i}\right)\right)\left(B \varphi_{i}, \varphi_{j}\right) \\
& =\frac{f\left(\lambda_{j}\right)-f\left(\lambda_{i}\right)}{\lambda_{j}-\lambda_{i}}\left(\lambda_{j}-\lambda_{i}\right)\left(B \varphi_{i}, \varphi_{j}\right) \\
& =\frac{f\left(\lambda_{j}\right)-f\left(\lambda_{i}\right)}{\lambda_{j}-\lambda_{i}}\left((A B-B A) \varphi_{i}, \varphi_{j}\right) .
\end{aligned}
$$


Now, a transformation similar to the passage from (3) to (5) yields

$$
f(A) B-B f(A)=\iint \frac{f(\lambda)-f(\mu)}{\lambda-\mu} d E_{\lambda} T d E_{\mu}
$$

where $T=A B-B A$.

We see that this formula (unlike (5)) only involves the spectral measure of $A$. Looking at formulas (8), (5) and (6) we note that an approximate equality may be written:

$$
f(A) B-B f(A) \cong(1 / i)[f(A+i(A B-B A))-f(A)] .
$$

Therefore it is natural to expect that the result obtained for the difference $f(B)-f(A)$ can be transferred to commutators in a suitable way. This was done by Birman and Solomyak [6] and by the author [14].

2. Kantorovich-Rubinshteĭn norm. Suppose the function $f$ defined on the real line satisfies the Lipschitz condition with a constant which we denote by $[f]$. The following approach was used mainly by the author. Take the basic formula of functional calculus in operator theory:

$$
f(A)=\int f(\lambda) d E_{\lambda} .
$$

For our applications, this formula has some disadvantage: it cannot be integrated by parts. So it is natural to examine the difference of operators, which leads at once to problems of perturbation theory:

$$
((f(A)-f(B)) x, x)=\int f(\lambda)\left(\left(d E_{\lambda}-d F_{\lambda}\right) x, x\right) .
$$

After integration by parts we get (the non-integral term is obviously zero)

$$
((f(A)-f(B)) x, x)=\int\left(\left(E_{\lambda}-F_{\lambda}\right) x, x\right) d f .
$$

Thus if $\|x\| \leq 1$ then

$\|f(A)-f(B)\|=\sup _{\|x\| \leq 1}\left|\int\left(\left(E_{\lambda}-F_{\lambda}\right) x, x\right) d f\right| \leq[f] \sup \int\left|\left(\left(E_{\lambda}-F_{\lambda}\right) x, x\right)\right| d \lambda$.

So we have

$$
\begin{aligned}
\sup _{[f] \leq 1}\|f(A)-f(B)\| & \leq \sup _{\|x\| \leq 1} \int\left|\left(\left(E_{\lambda}-F_{\lambda}\right) x, x\right)\right| d \lambda \\
& =\sup _{\|x\| \leq 1}\left\|\left(\left(E_{\lambda}-F_{\lambda}\right) x, x\right)\right\|_{L_{1}} .
\end{aligned}
$$

Note that in fact there is equality in the last formula (take $f$ with $f^{\prime}(t)=$ $\left.\operatorname{sign}\left(\left(E_{t}-F_{t}\right) x, x\right)\right)$.

Formula (9) was first obtained many years ago with the help of the K-R norm. We briefly describe this approach here as it seems to be of use.

Consider the set function

$$
((E(\Delta)-F(\Delta)) x, x)=\Phi_{x}(\Delta)
$$


with fixed $x$. If $A, B$ are bounded operators then there exists an interval $[a, b]=K$ on the real line which contains the support of the function $\Phi_{x}(\Delta)$. Since $\Phi_{x}(K)=$ $\|x\|^{2}-\|x\|^{2}=0$, we can define the K-R norm for $\Phi_{x}(\Delta)$. We do not give the precise definition; let us just say that the K-R norm (in our case) is the minimal work for redistribution of the mass of size $\|x\|^{2}$ originally distributed by the law $(E(\Delta) x, x)$ to the law $(F(\Delta) x, x)$. So for spectral measures we define

$$
\|E-F\|_{\mathrm{K}-\mathrm{R}}=\sup _{\|x\| \leq 1}\left\|\Phi_{x}(\Delta)\right\|_{\mathrm{K}-\mathrm{R}} .
$$

Since the function space Lip 1 with norm $[f]$ (functions differing by a constant being regarded as identical) is dual to the $K-\mathrm{R}$ space, using the general form of a linear functional on the K-R space we obtain

$$
\begin{aligned}
\|((E(\Delta)-F(\Delta)) x, x)\|_{\mathrm{K}-\mathrm{R}} & =\sup _{[f] \leq 1}\left|\int \Phi_{x}(\Delta) d f\right| \\
& =\sup _{[f] \leq 1}\left|\int f(\lambda) d\left(\left(E_{\lambda}-F_{\lambda}\right) x, x\right)\right| \\
& =\sup _{[f] \leq 1}|((f(A)-f(B)) x, x)| .
\end{aligned}
$$

Taking the supremum over $x$ we get

$$
\begin{aligned}
\|E-F\|_{\mathrm{K}-\mathrm{R}} & =\sup _{\|x\| \leq 1}\|((E(\Delta)-F(\Delta)) x, x)\| \\
& =\sup _{[f] \leq 1} \sup _{\|x\| \leq 1} \mid((f(A)-f(B)) x, x \mid \\
& =\sup _{[f] \leq 1}\|f(A)-f(B)\| .
\end{aligned}
$$

Since in the one-dimensional case the K-R norm coincides with the $L_{1}$ norm, we again obtain (9).

First the K-R norm for spectral functions was introduced by F. Kunert [19] (in that work the connection between the K-R norm and functions of operators was not settled yet). The idea of applying this norm to spectral distributions is due to the late Prof. M. K. Gavurin who was the thesis supervisor of both F. Kunert and the present author.

We can obtain the following formula for commutators by analogy with (9):

$$
\begin{aligned}
\sup _{[f] \leq 1}\|f(A) B-B f(A)\| & =\sup _{\|x\| \leq 1} \int\left|\left(\left(E_{\lambda} B-B E_{\lambda}\right) x, x\right)\right| d \lambda \\
& =\sup _{\|x\| \leq 1}\left\|\left(\left(E_{\lambda} B-B E_{\lambda}\right) x, x\right)\right\|_{\mathrm{K}-\mathrm{R}} \\
& =\left\|E_{\lambda} B-B E_{\lambda}\right\|_{\mathrm{K}-\mathrm{R}} .
\end{aligned}
$$

We can drop the assumption that $B$ is a selfadjoint operator if we modify the latter formula by replacing the second $x$ in the inner product by $y$ and take the supremum over all $x, y$ with $\|x\| \leq 1,\|y\| \leq 1$, using the equality $(E(K) B x, y)-$ $(B E(K) x, y)=(B x, y)-(B x, y)=0$. 
The principal results for the operator norm obtained in [12] and [14] for $f \in$ Lip 1 with Lipschitz constant $[f] \leq 1$ are:

$$
\begin{gathered}
\|f(A)-f(B)\| \leq C_{1}\left(\log \frac{\|A\|+\|B\|}{\|A-B\|}+2\right)^{2}\|A-B\|, \\
\|f(A) B-B f(A)\| \leq C_{2}\left[\log \left(\frac{4\|A\|\|B\|}{\|A B-B A\|}+1\right)+2\right]^{2}\|A B-B A\| .
\end{gathered}
$$

More generally, for $f \in \operatorname{Lip} \alpha$, where $0<\alpha \leq 1$, we can derive

$$
\begin{aligned}
\|f(A)-f(B)\| & \leq C_{3}\left(\log \frac{\|A\|+\|B\|}{\|A-B\|}+2\right)^{2 \alpha}\|A-B\|^{\alpha}, \\
\|f(A) B-B f(A)\| & \leq C_{4}\left[\log \left(\frac{4\|A\|\|B\|}{\|A B-B A\|}+1\right)+2\right]^{2 \alpha}\|A B-B A\|^{\alpha} .
\end{aligned}
$$

The estimate (13) can be compared with the result in [2] for fractional powers:

$$
\left\|A^{\alpha}-B^{\alpha}\right\| \leq\|A-B\|^{\alpha},
$$

where $A, B$ and $A-B$ are positive operators.

Let us return to the discussion of Lip 1 functions. An important example of such a function is $f(x)=|x|$. In [16], T. Kato obtained directly the estimate

$$
\||A|-|B|\| \leq \frac{2}{\pi}\left(\log \frac{\|A\|+\|B\|}{\|A-B\|}+2\right)\|A-B\| .
$$

In [12] the author constructed an example showing the impossibility of a linear estimate between $\|f(A)-f(B)\|$ and $\|A-B\|$. Incidentally, this can also be easily derived from the example constructed by McIntosh in [20]. Methods of construction of such type examples are also discussed in [21] and in Section 3 of the present paper.

E. B. Davies [10] obtained the following inequality for commutators in the Schatten ideals $\mathfrak{S}_{p}$ :

$$
\|f(A) B-B f(A)\|_{p} \leq C_{5}\left(1+\alpha_{p}\right)\|A B-B A\|_{p}
$$

for $1<p<\infty$, and calculated the constant $\alpha_{p}$ explicitly.

Now we discuss the possibility of passing to normal operators. F. Kittaneh [17] obtained an estimate of the Hilbert-Schmidt norm for normal operators. To be more precise, if $N$ is a normal operator and if the commutator $N X-X N$ belongs to the Hilbert-Schmidt class (i.e. it is a compact operator and the sum of the squares of its singular values is convergent), then

$$
\|f(N) X-X f(N)\|_{2} \leq[f]\|N X-X N\|_{2} \text {. }
$$

In [13] the following estimate is stated. Let $A_{1}, A_{2}$ be a pair of commuting bounded selfadjoint operators, $B_{1}, B_{2}$ be another such pair, and $f(x, y)$ be a real function defined in a rectangle whose projections on the real and imaginary axes 
contain the spectra of the operators $A_{1}, A_{2}$ and $B_{1}, B_{2}$, respectively. Suppose $f$ satisfies the Lipschitz condition with constant $[f]$ in this rectangle. Then

$$
\begin{aligned}
& \left\|f\left(A_{1}, A_{2}\right)-f\left(B_{1}, B_{2}\right)\right\| \\
\leq & {[f] C_{6}\left(\log \frac{\left\|A_{1}\right\|+\left\|A_{2}\right\|+\left\|B_{1}\right\|+\left\|B_{2}\right\|}{\left\|A_{1}-B_{1}\right\|+\left\|A_{2}-B_{2}\right\|}+C_{7}\right)^{3}\left(\left\|A_{1}-B_{1}\right\|+\left\|A_{2}-B_{2}\right\|\right) . }
\end{aligned}
$$

The proof of this estimate is based on the proof of (11) and is rather involved. Moreover, the use of the K-R norm is essential here.

The question arises of whether the estimates (11), (12) or (16) carry over to normal operators. The following difficulties arise in this case: first, the spectrum of a normal operator lies in the complex plane (and not only on the real line as for a selfadjoint operator), which complicates proofs considerably. Secondly, the methods of Birman and Solomyak lead to consideration of much more smooth functions, namely to analytic functions.

Note that the estimate (11) does hold for normal operators. This was proved by the author in [14]. The examination of commutators is more complicated. R. Moore [22] proved in 1976 that if $A B-B A$ is "small", then $A^{*} B-A B^{*}$ is also "small". ( $A^{*}$ is the adjoint operator to $A$.) If $A B-B A$ and $A^{*} B-A B^{*}$ are known to be "small" it is easy to obtain the following estimate:

$$
\|f(A) B-B f(A)\| \leq C_{8}\left(\log \frac{2\|A\|+2\|B\|}{K}+C_{9}\right)^{3}[f] K
$$

where

$$
K=\|A B-B A\|+\left\|A^{*} B-B A^{*}\right\|, \quad[f]=\sup \frac{\left|f(z)-f\left(z^{\prime}\right)\right|}{\left|z-z^{\prime}\right|} .
$$

But the question is how to estimate the norm $\|f(A) B-B f(A)\|$ by $\|A B-B A\|$ only, without using $\left\|A^{*} B-B A^{*}\right\|$.

It is clear that we should estimate the norm of $A^{*} B-B A^{*}=f(A) B-B f(A)$ where $f(z)=\bar{z}$. The following estimate may be obtained locally:

$$
\left\|A^{*} B-B A^{*}\right\| \leq C_{10}\|A B-B A\|^{1 / 2} .
$$

This estimate is fairly straightforward (similar to the selfadjoint case) and comes from the following informal consideration.

Let us examine normal matrices in the $n$-dimensional space once more and return to formula (8). For $f(z)=\bar{z}$ and $\lambda_{i} \neq \lambda_{j}$ this formula leads to

$$
\left(\left(A^{*} B-B A^{*}\right) \varphi_{i}, \varphi_{j}\right)=\frac{\bar{\lambda}_{i}-\bar{\lambda}_{j}}{\lambda_{i}-\lambda_{j}}\left((A B-B A) \varphi_{i}, \varphi_{j}\right) .
$$

Obviously, if $\lambda_{i}=\lambda_{j}$ then $\left(\left(A^{*} B-B A^{*}\right) \varphi_{i}, \varphi_{j}\right)=\left((A B-B A) \varphi_{i}, \varphi_{j}\right)=0$.

Therefore, the elements of the matrix of $A^{*} B-B A^{*}$ in the basis formed by the eigenvectors of $A$ are the corresponding elements of $A B-B A$ multiplied by numbers of modulus one. It is clear that the norm may increase under this operation not more than $n^{1 / 2}$ times. With the help of the Weyl-von Neumann 
lemma (see [1], p. 322) and of another lemma of the author (see, for example, [14], p. 239), $n$ can always be replaced by $2(\|A\|+\|B\|) /\|A-B\|$, and (17) follows. But it seems to the author that an estimate of type (12) holds for $f(z)=\bar{z}$ as well.

To end this section, we indicate the proof of estimate (11) and its relation to DOI.

First, let us formulate the main lemma proved in detail in [12]. Let $T$ be a selfadjoint bounded operator in $H,\left\{\varphi_{i}\right\}$ and $\left\{\psi_{i}\right\}$ be two systems of vectors in $H$, where the non-zero vectors from $\left\{\varphi_{i}\right\}$ form an orthonormal system. Moreover, let $\lambda_{1}<\ldots<\lambda_{n}$ be real numbers, and $x=\left\{x_{i}\right\}$ and $y=\left\{y_{i}\right\}(i=1, \ldots, n)$ be vectors in $\mathbb{C}^{n}$, with $\|x\|=\left(\sum_{i}\left|x_{i}\right|\right)^{1 / 2} \leq 1$ and $\|y\| \leq 1$. Then

$$
\sum_{i=1}^{n}\left(\lambda_{i+1}-\lambda_{i}\right)\left|\sum_{k=1}^{i} \sum_{j=i+1}^{n} x_{i} \bar{y}_{j} \frac{\left(T \varphi_{i}, \psi_{j}\right)}{\lambda_{j}-\lambda_{i}}\right| \leq \log n(\log n+1)\|T\| .
$$

Having this inequality we can replace $n$ in the right hand side by $(\|A\|+$ $\|B\|) /\|A-B\|$, and the whole left hand side by $\|f(A)-f(B)\|$ as noted above. However, in the process some constants appear (as we think, due to the imperfection of the proof), depending on the type of problems to which this lemma is used.

On the other hand, if $A$ and $B$ are selfadjoint operators in $H$ and $f$ is a Lipschitz function on the real line, we can write the quadratic form $(((f) A)-$ $f(B)) x, y)$ with the help of DOI which becomes an ordinary double Stieltjes integral in this case:

$$
\left(((f(A)-f(B)) x, y)=\iint \frac{f(\lambda)-f(\mu)}{\lambda-\mu}\left(d F_{\mu}(A-B) d E_{\lambda} x, y\right) .\right.
$$

Let us split the domain of integration into two parts: $\lambda<\mu$ and $\lambda \geq \mu$, and write $f(\lambda)-f(\mu)=\int_{\mu}^{\lambda} f^{\prime}(\nu) d \nu$. We obtain the following representation:

$$
\begin{aligned}
(((f(A)-f(B)) x, y)= & \int_{-\infty}^{\infty} d f(\nu) \int_{-\infty}^{\nu} \int_{\nu}^{\infty} \frac{\left(d F_{\mu}(A-B) d E_{\lambda} x, y\right)}{\lambda-\mu} \\
& +\int_{-\infty}^{\infty} d f(\nu) \int_{\nu}^{\infty} \int_{-\infty}^{\nu} \frac{\left(d F_{\mu}(A-B) d E_{\lambda} x, y\right)}{\lambda-\mu}
\end{aligned}
$$

An obvious estimate gives

$$
\begin{aligned}
|((f(A)-f(B)) x, y)| \leq[f] & \left\{\int_{-\infty}^{\infty}\left|\int_{-\infty}^{\nu} \int_{\nu}^{\infty} \frac{\left(d F_{\mu}(A-B) d E_{\lambda} x, y\right)}{\lambda-\mu}\right| d \nu\right. \\
& \left.+\int_{-\infty}^{\infty}\left|\int_{\nu}^{\infty} \int_{-\infty}^{\nu} \frac{\left(d F_{\mu}(A-B) d E_{\lambda} x, y\right)}{\lambda-\mu}\right| d \nu\right\} .
\end{aligned}
$$

Now it is clear that the left hand side of (18) is a partial sum for the first of these integrals, where $T=A-B$. We believe that the integral analogue of inequality (18) can be proved by using the Helly theorem and the theory of double 
Stieltjes integrals but this is not yet checked in detail. This inequality could be written as follows:

$$
\int_{-\infty}^{\infty} d \nu\left|\int_{-\infty}^{\nu} \int_{\nu}^{\infty} \frac{\left(d F_{\mu}(A-B) d E_{\lambda} x, y\right)}{\lambda-\mu}\right| \leq \log P(\log P+1)\|A-B\|
$$

where $P=(\|A\|+\|B\|) /\|A-B\|$.

We also wish to formulate the most difficult point of the possible proof, which is the exact integral analogue of Lemma 7 of [12], p. 154.

Let $\Phi(\lambda, \mu)$ be a real function of bounded variation defined in the square $a \leq \lambda \leq b, a \leq \mu \leq b$, let $a \leq p<q \leq b$ and assume

$$
\int_{a}^{p} \int_{q}^{b} \frac{d^{2} \Phi(\lambda, \mu)}{\mu-\lambda}>0
$$

Then there exists a non-increasing function $g(\lambda)$ such that

$$
\int_{a}^{p} \int_{q}^{b} \frac{p-\lambda}{\mu-\lambda} d^{2} \Phi(\lambda, \mu) \leq \int_{a}^{p} \int_{q}^{g(\lambda)} d^{2} \Phi(\lambda, \mu) .
$$

Note that this estimate has no absolute value signs.

We think that the rigorous proof of the results of [12] in terms of integrals (but not of sums) could simplify them and lead to decreasing the relevant constants.

\section{Some unsolved problems}

1. Let $\mathbb{R}^{n}$ be the real Euclidean space and let $A$ be a real diagonal matrix, $A=\operatorname{diag}\left\{\lambda_{i}\right\}$. Assume for simplicity that the eigenvalues of $A$ are distinct, $\lambda_{1}<$ $\ldots<\lambda_{n}$.

Let $B$ be a real symmetric matrix and $T=B-A$. Consider a certain family $\{S\}$ of matrices which may differ from $T$ in the signs of blocks on the main diagonal.

The family $\{S\}$ is constructed in the following way. Let $\left\{\varphi_{i}\right\}$ be a system of eigenvectors of $A$ where $\varphi_{i}$ corresponds to the eigenvalue $\lambda_{i}$. Let $P$ be the orthogonal projector on $\operatorname{span}\left\{\varphi_{i}\right\}_{i=1}^{k}, k=1, \ldots, n-1$. Then it is obvious that $T$ may be represented as

$$
T=P T P+(I-P) T(I-P)+P T(I-P)+(I-P) T P
$$

(this representation holds for any projector $P$ ).

At the first stage we include two matrices in the family $\{S\}: S_{1}=T$ and

$$
S_{2}=P T P+(I-P) T(I-P)-P T(I-P)-(I-P) T P .
$$

Next, for $k>1$, consider the matrix $T_{1}=P T P$. Choose $k_{1}$ with $1 \leq k_{1} \leq k-1$ and consider the projector $P_{1}$ on span $\left\{\varphi_{i}\right\}_{i=1}^{k_{1}}$. (It is clear that $P_{1}=P_{1} P$.) Then

$$
T_{1}=P_{1} T_{1} P_{1}+\left(I-P_{1}\right) T_{1}\left(I-P_{1}\right)+\left(I-P_{1}\right) T_{1} P_{1}+P_{1} T_{1}\left(I-P_{1}\right) .
$$


We also consider the matrix $T_{2}$ which differs from $T_{1}$ in the signs of the last two terms:

$$
T_{2}=P_{1} T_{1} P_{1}+\left(I-P_{1}\right) T_{1}\left(I-P_{1}\right)-\left(I-P_{1}\right) T_{1} P_{1}-P_{1} T_{1}\left(I-P_{1}\right) .
$$

We include in $\{S\}$ the two matrices obtained from (19) and (20) by replacing $P T P$ by $T_{2}$ :

$$
\begin{aligned}
& S_{3}=T_{2}+(I-P) T(I-P)+(I-P) T P+P T(I-P), \\
& S_{4}=T_{2}+(I-P) T(I-P)-P T(I-P)-(I-P) T P .
\end{aligned}
$$

If $k=1$ at the first stage, we take $T_{2}=-P T P$.

We continue this process as long as possible.

Similarly we transform the matrix $(I-P) T(I-P)$. (Here we choose the projector on $\operatorname{span}\left\{\varphi_{k+1}, \ldots, \varphi_{r}\right\}$, where $1 \leq r \leq n-1$.) Note that all matrices in the family $\{S\}$ are symmetric and real.

We make the following conjecture:

For any function $f \in \operatorname{Lip} 1,[f] \leq 1$, defined on an interval which contains the spectra of the operators $A, A+T$ in its interior, there exists a matrix $S_{0}$ in the family $\{S\}$ such that

$$
\|f(A+T)-f(A)\| \leq\left\|S_{0}\right\| .
$$

(A similar inequality might also be true for $\mathfrak{S}_{p}$ norms.)

Note that a converse assertion (in some sense) can be proved: for any matrix $S_{1}$ in the family $\{S\}$ there exist a diagonal matrix $A$ and a function $f$ with $[f] \leq 1$ such that $\|f(A+T)-f(A)\|$ is as close to $\left\|S_{1}\right\|$ as we wish. This allows one to construct various counterexamples.

2. Let $f$ be a function in Zygmund's class on the real line, i.e. $\| f(t+h)+$ $f(t-h)-2 f(t) \| \leq c h$ where $c$ is a constant. Does the estimate

$$
\|f(A+T)+f(A-T)-2 f(A)\| \leq c_{1}\|T\|
$$

hold? (Of course $A$ and $T$ are bounded selfadjoint operators.)

3. It would be interesting to obtain estimates of type (11), (12) with the help of the theory of functions of a complex variable. E. M. Dynkin believes that at least Kato's estimate for $f(x)=|x|$ can be obtained in this way.

4. The problem of estimating $\left\|A^{*} B-B A^{*}\right\|$ in terms of $\|A B-B A\|$ seems to be of great importance.

We also wish to mention another two problems studied in the literature, which may be closely related to the counterexamples obtained for the purpose of our main topic.

5. Characterize the selfadjoint operators $A, B$ such that the trace formula (7) holds for any $f \in \operatorname{Lip} 1$. Is the condition " $E_{\lambda}-F_{\lambda} \in \mathfrak{S}_{1}$ for each $\lambda$ " relevant here?

6. The Bourgain problem. Let an operator $A$ be similar to a contraction in the $n$-dimensional space, i.e. there exists a non-singular matrix $C$ such that $\left\|C^{-1} A C\right\| \leq 1$ (this means that the eigenvalues of $A$ do not exceed 1). Suppose also that $\left\|A^{k}\right\| \leq M$ for any $k$. J. Bourgain proved [7] that in this case the 
matrix $C$ can be chosen so that $\|C\| \cdot\left\|C^{-1}\right\| \leq C_{11} \log n$. The problem is to prove or disprove the existence of $C$ satisfying $\|C\| \cdot\left\|C^{-1}\right\| \leq K$ where $K$ is a constant independent of the dimension $n$.

7. Approximation of almost commuting operators (especially selfadjoint) by commuting pairs (see [8] and [26]).

In conclusion the author is grateful to E. M. Dynkin, N. K. Nikol'skiu and G. V. Rosenblum for very useful discussions of this article.

\section{References}

[1] N. I. Akhiezer and I. M. Glazman, Theory of Linear Operators in Hilbert Space, Nauka, Moscow, 1966 (in Russian).

[2] M. Sh. Birman, L. S. Koplienko and M. Z. Solomyak, Estimates of the spectrum of the difference of fractional powers of selfadjoint operators, Izv. Vyssh. Uchebn. Zaved. Mat. 1975 (3) (154), 3-10 (in Russian)

[3] M. Sh. Birman and M. Z. Solomyak, Double Stieltjes operator integrals, in: Probl. Mat. Fiz. 1, Leningrad Univ., 1966, 33-67 (in Russian).

[4] - - - Double Stieltjes operator integrals II, in: Probl. Mat. Fiz. 2, Leningrad Univ., 1967, 26-60 (in Russian).

[5] —, - Remarks on spectral shift functions, Zap. Nauchn. Sem. LOMI 27 (1972), 33-41 (in Russian).

[6] - - - Operator integration, perturbation and commutators, ibid. 170 (1989), 34-66 (in Russian).

[7] J. Bourgain, On the similarity problem for polynomially bounded operators on Hilbert space, Israel J. Math. 54 (1986), 224-241.

[8] M. D. Choi, Almost commuting matrices need not be nearly commuting, Proc. Amer. Math. Soc. 102 (1988), 529-533.

[9] Yu. L. Daletskiŭ and S. G. Kreŭn, Formulas for differentiation with respect to parameters of functions of hermitian operators, Dokl. Akad. Nauk SSSR 76 (1951), 13-16 (in Russian).

[10] E. B. Davies, Lipschitz continuity of functions of operators in the Schatten classes, J. London Math. Soc. 37 (1988), 148-157.

[11] Yu. B. Farforovskaya, An example of a Lipschitz function of a selfadjoint operator giving a non-nuclear increment under a nuclear perturbation, Zap. Nauchn. Sem. LOMI 39 (1974), 194-195 (in Russian).

[12] - , An estimate of the norm $\|f(A)-f(B)\|$ for selfadjoint operators $A$ and $B$, ibid. 56 (1976), 143-162 (in Russian).

[13] - An estimate of the norm $\left\|f\left(A_{1}, A_{2}\right)-f\left(B_{1}, B_{2}\right)\right\|$ for pairs of commuting selfadjoint operators, ibid. 135 (1984), 175-177 (in Russian).

[14] -, Commutators of functions of operators in perturbation theory, in: Probl. Mat. Anal. 12 (1992), 234-247 (in Russian).

[15] L. V. Kantorovich and G. Sh. Rubinshteŭn, On a space of completely additive functions, Vestnik Leningrad. Gos. Univ. 13 (7) (1958), 52-59 (in Russian).

[16] T. Kato, Continuity of the map $S \rightarrow|S|$ for linear operators, Proc. Japan Acad. 49 (1973), 157-160.

[17] F. Kittaneh, On Lipschitz functions of normal operators, Proc. Amer. Math. Soc. 94 (1985), 416-418.

[18] M. G. Kreŭn, On a trace formula in perturbation theory, Mat. Sb. 33 (1953), 597-626 (in Russian). 
[19] F. Kunert, The Kantorovich-Rubinshteĭn metric and convergence of selfadjoint operators, Vestnik Leningrad. Gos. Univ. 20 (13) (3) (1965), 37-49 (in Russian).

[20] A. McIntosh, Counterexample to a question on commutators, Proc. Amer. Math. Soc. 29 (1971), 337-340.

[21] B. Mirman, A source of counterexamples in operator theory and how to construct them, Linear Algebra Appl. 169 (1992), 49-59.

[22] R. Moore, An asymptotic Fuglede theorem, Proc. Amer. Math. Soc. 50 (1975), 138-142.

[23] V. V. Peller, Hankel operators and differentiability properties of functions of selfadjoint (unitary) operators, preprint LOMI, Leningrad, 1984.

[24] - , Hankel operators in the theory of perturbations of unitary and selfadjoint operators, Funktsional. Anal. i Prilozhen. 19 (2) (1985), 37-51 (in Russian).

[25] -, For which $f$ does $A-B \in \mathfrak{S}_{p}$ imply that $f(A)-f(B) \in \mathfrak{S}_{p}$ ?, in: Oper. Theory: Adv. Appl. 24, Birkhäuser, 1987, 289-294.

[26] D. Voiculescu, Asymptotically commuting finite rank unitary operators without commuting approximation, Acta Sci. Math. (Szeged) 45 (1983), 429-431. 